\title{
A facile access and evaluation of some novel thiazole and 1,3,4-thiadiazole derivatives incorporating thiazole moiety as potent anticancer agents
}

Sobhi M. Gomha ${ }^{1 *} \mathbb{D}$, Mohamad R. Abdelaziz², Nabila A. Kheder ${ }^{1,3}$, Hassan M. Abdel-aziz ${ }^{4}$, Seham Alterary ${ }^{5}$ and Yahia N. Mabkhot ${ }^{5^{*}}$

\begin{abstract}
Background: Many heterocyclic compounds containing thiazole or 1,3,4-thiadiazole ring in their skeletons have been reported to possess various pharmacological activities especially anticancer activities.

Results: 4-Methyl-2-phenylthiazole-5-carbohydrazide (2) was used as a synthon to prepare 2-(4-methyl-2-phenylthiazole-5-carbonyl)-N-phenylhydrazinecarbothioamide (3) and 2-(2-(4-methyl-2-phenylthiazole-5-carbonyl) hydrazono)- $N^{\prime}$-phenylpropane hydrazonoyl chlorides $\mathbf{5 a}-\mathbf{c}$. In addition, thioamide $\mathbf{3}$ was used as starting material for preparation of a new series of thiadiazole derivatives via its reaction with hydrazonoyl chlorides $\mathbf{5 a} \mathbf{a}-\mathbf{c}$ in dioxane using triethylamines as catalyst. In addition, a series of thiazole derivatives was synthesized by reaction of thioamide $\mathbf{3}$ with a number of a-halo compounds, namely, 3-chloropentane-2,4-dione (8) or 2-chloro-3-oxo-N-phenyl butanamide (10) phenacyl bromide $\mathbf{1 2}$ ethyl chloroacetate (14) in $\mathrm{EtOH}$ in the presence of triethylamine. The structures assigned for all the new products were elucidated based on both elemental analyses and spectral data and the mechanisms of their formation was also discussed. Moreover, the new products was evaluated in vitro by MTT assays for their anticancer activity against cell lines of Hepatocellular carcinoma cell line (HepG-2). The best result observed for compounds $\mathbf{7 b}$ $\left(I C_{50}=1.61 \pm 1.92(\mu \mathrm{g} / \mathrm{mL})\right)$ and $\mathbf{1 1}\left(\mathrm{IC}_{50}=1.98 \pm 1.22(\mu \mathrm{g} / \mathrm{mL})\right)$. The structure-activity relationships have been suggested based on their anticancer results.
\end{abstract}

Conclusions: A novel series of new pharmacophores containing thiazole moiety have been synthesized using a facile and convenient methods and evaluated as potent anticancer agents.

Keywords: Thiazoles, Thiadiazoles, Hydrazonoyl chlorides, Phenacyl bromide, Thioamide, Anticancer activity

\section{Introduction}

Identification of novel structure leads that may be of use in designing new, potent, selective and less toxic anticancer agents remains a major challenge for medicinal chemistry researchers. Compounds containing thiazole core have diverse biological activities as antihypertension,

\footnotetext{
*Correspondence: s.m.gomha@gmail.com; yahia@ksu.edu.sa ${ }^{1}$ Department of Chemistry, Faculty of Science, Cairo University, Giza 12613, Egypt

${ }^{5}$ Department of Chemistry, College of Science, King Saud University, P. O. Box 2455, Riyadh 11451, Saudi Arabia

Full list of author information is available at the end of the article
}

antifungal, antimicrobial, anti-inflammatory, antioxidant, antitubercular [1-7], and anticancer [8-12]. Also, thiazole ring present in many drugs such as Nizatidine, Abafungin, and Amiphenazole (Fig. 1).

Many biological activities were reported for the compounds containing 1,3,4-thiadiazole ring such as antituberculosis, anti-inflammatory, antidepressant and anxiolytic, antioxidant, anticonvulsants [13-17] and anticancer activities [18-20]. In addition, many drugs containing 1,3,4-thiadiazole ring are available in the market such as acetazolamide, methazolamide, and megazol (Fig. 2). 
In continuation of our studies dealing with the utility of hydrazonoyl halides for synthesis of various bioactive bridgehead nitrogen polyheterocycles [21-30], we wish to report herein a new facile synthesis of new heterocycles containing thiazole and 1,3,4-thiadiazole or two thiazole rings in one molecular frame. We anticipated that the synthesized compounds would have potent pharmacological activities.

\section{Results and discussion}

\section{Chemistry}

2-(4-Methyl-2-phenylthiazole-5-carbonyl)- $N$-phenylhydrazinecarbothioamide (3) [31] was prepared via reaction of 4-methyl-2-phenylthiazole-5-carbohydrazide (2) with phenyl isothiocyanate in EtOH (Scheme 1).

The reaction of compound 2 with the appropriate hydrazonoyl chlorides $\mathbf{4 a - c}$ [32] in refluxing ethanol yielded the corresponding condensation product 5 (Scheme 2). The IR spectra of the latter products revealed a carbonyl and two $\mathrm{NH}$ absorption bands (see "Experimental" part). Their ${ }^{1} \mathrm{HNMR}$ showed two $\mathrm{D}_{2} \mathrm{O}$ exchangeable signals of two $\mathrm{NH}$ protons in the regions $\delta 10.03-10.06$ and $\delta 10.57-10.59 \mathrm{ppm}$. Also, their mass spectra confirmed the assigned structure 5 (Scheme 2). Treatment of thioamide derivative 3 with the appropriate hydrazonoyl halides of type $\mathbf{5 a}-\mathbf{c}$ in refluxing $\mathrm{EtOH}$<smiles>CN/C(=C\[N+](=O)[O-])NCCSCc1csc(CN(C)C)n1</smiles>

Nizatidine

treatment of peptic ulcer and gastroesophageal reflux diseases

Fig. 1 Some marketed drugs containing thiazole ring<smiles>Cc1ccc(Oc2ccccc2-c2csc(NC3=NCCCN3)n2)c(C)c1</smiles>

Abafungin

treatment of dermatomycoses

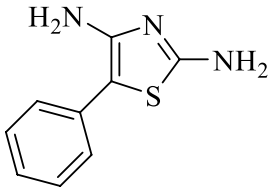

Amiphenazole

used as an antidote for barbiturate or opiate overdose<smiles>CC(=O)Nc1nnc(S(N)(=O)=O)s1</smiles>

Acetazolamide

Treat glaucoma, epilepsy, altitude sickness, periodic paralysis, and heart failure<smiles>CC(=O)/N=c1/sc(S(N)(=O)=O)nn1C</smiles>

Methazolamide

potent carbonic anhydrase inhibitor<smiles>C[14C](=[W])O[N+](=O)c1cnc(-c2nnc(N)s2)n1C</smiles>

cures some protozoan infections

Fig. 2 Examples of drugs containing a 1,3,4-thiadiazole ring<smiles>CCOC(=O)c1sc(-c2ccccc2)nc1CNNc1sc(-c2ccccc2)nc1C</smiles>

Scheme 1 Synthesis of thiazoles $\mathbf{2 , 3}$ 


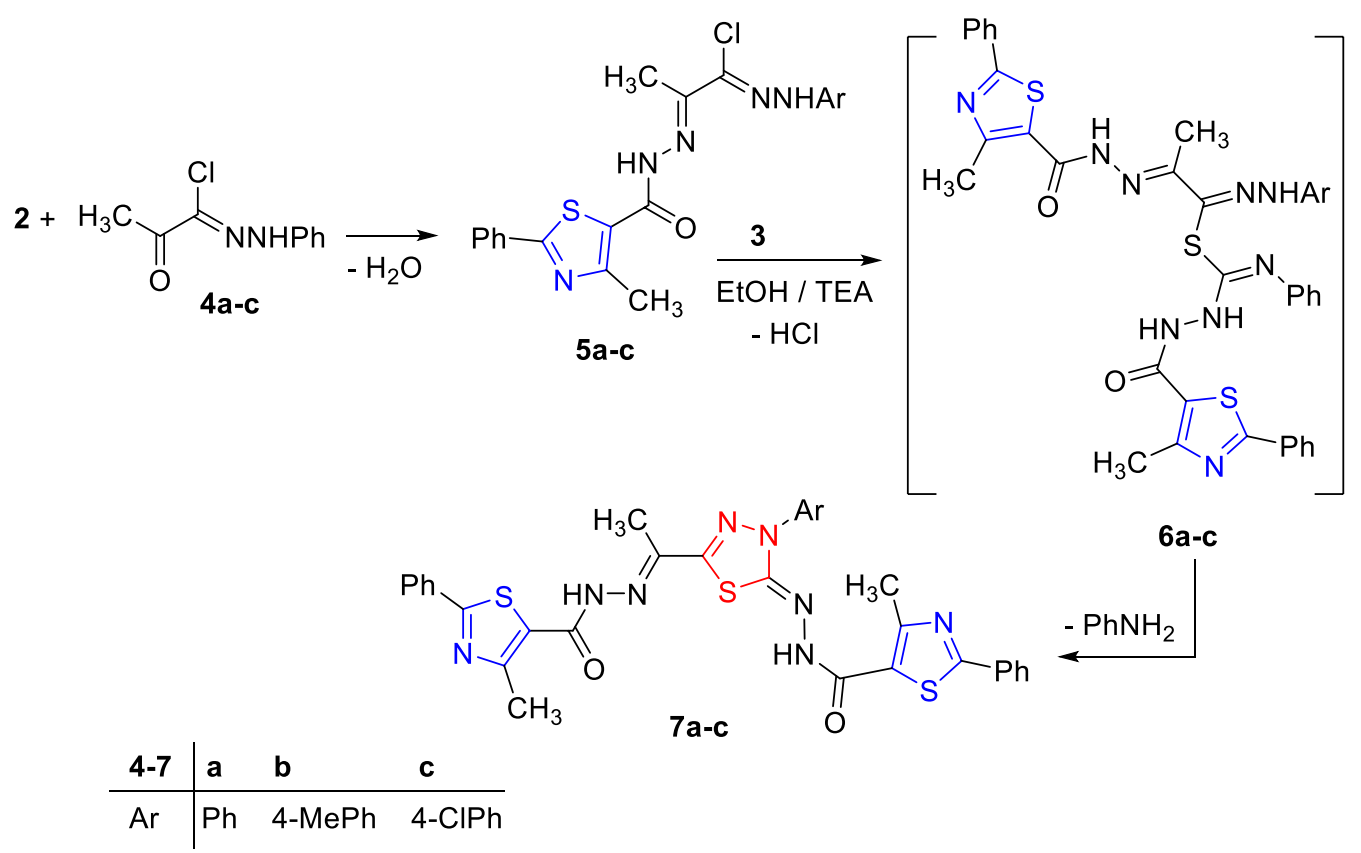

Scheme 2 Synthesis of thiadiazole derivatives $\mathbf{7 a - c}$

containing TEA gave the corresponding thiadiazole derivatives $7 \mathbf{a}-\mathbf{c}$ (Scheme 2). Their structures were elucidated on the basis of their spectral data and elemental analysis (see "Experimental").

Next, refluxing of compound 3 with 3-chloropentane2,4-dione (8) or 2-chloro-3-oxo- $N$-phenyl butanamide (10) in $\mathrm{EtOH}$ in the presence of triethylamine afforded the thiazole derivatives 9 and 11, respectively (Scheme 3).The structure of compounds $\mathbf{9}$ and $\mathbf{1 1}$ were elucidated based on their elemental analysis and spectral data (see "Experimental").

In a similar manner, thioamide 3 reacted with phenacyl bromide 12 under the same experimental condition to afford one isolable product 13 named as $N^{\prime}$-(3,4-diphenylthiazol2(3H)-ylidene)-4-methyl-2-phenyl thiazole-5-carbohydrazide (Scheme 3). The structure of thiazole 13 was established based on its elemental analysis and spectral data (see "Experimental").

Finally, thioamide derivative $\mathbf{3}$ reacted with ethyl chloroacetate (14) to afford thiazole $\mathbf{1 5}$ as showed in Scheme 3. Its IR spectrum showed absorption bands at v $3331(\mathrm{NH})$, and 1726, $1648(2 \mathrm{C}=\mathrm{O}) \mathrm{cm}^{-1}$. In addition, its ${ }^{1} \mathrm{HNMR}$ spectrum showed singlet signal at $\delta 4.23 \mathrm{ppm}$ due to the thiazolidinone $\left(\mathrm{CH}_{2}\right)$ group.

\section{Anticancer activity}

The synthesized compounds were tested as anticancer agents against human Hepatocellular carcinoma cell line (HepG-2) using colorimetric MTT assay. We also included the well-known anticancer standard drug
(Cisplatin) in the same assay to compare the potency of the synthesized compounds. The $\mathrm{IC}_{50}$ (the concentration of test compounds required to kill $50 \%$ of cell population) was determined (Table 1, Fig. 3).

The results of Table 1 revealed that the ascending order of the cytotoxic activity of the newly synthesized compounds towards the human Hepatocellular carcinoma cell line (HepG-2) were as follow: $\mathbf{5 c}<\mathbf{1 3}<\mathbf{5 a}<\mathbf{5 b}<\mathbf{9}<$ $\mathbf{7 c}<\mathbf{1 5}<\mathbf{7 a}<\mathbf{1 1}<\mathbf{7 b}$ (Fig. 4).

From the data of Table 1, we concluded the following structure-activity relationships (SARs):

- The thiazole ring is essential for the activity.

- Less number of thiazole ring as in compounds $\mathbf{5 a - c}$ lead to drastic drop in activity.

- 1,3,4-Thiadiazole ring is crucial for the cytotoxic activity.

- Presence of methyl group (electron donating group) at position 4 of the phenyl ring in compound $\mathbf{7 b}$ increase its activity more than compound $7 \mathbf{a}$.

- The presence of the N-phenylcarboxamide group in compound $\mathbf{1 1}$ leads to increasing of its cytotoxic activity.

\section{Experimental \\ Chemistry \\ General}

Melting points were measured on an Electrothermal IA 9000 series digital melting point apparatus (Bibby Sci. 


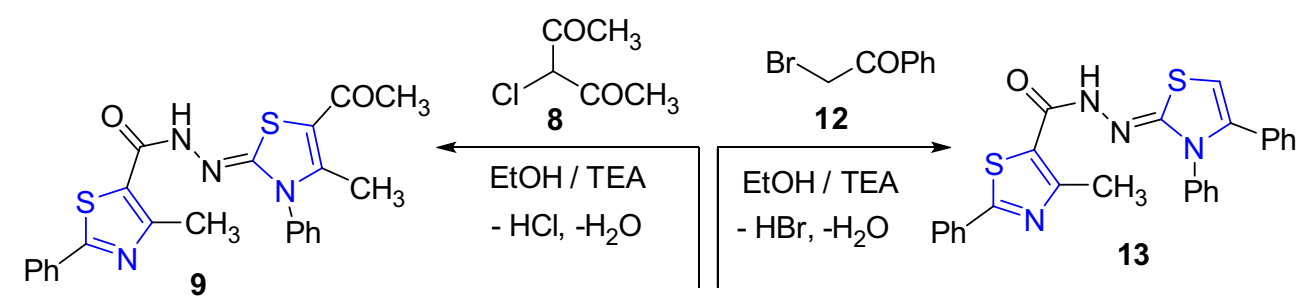<smiles>Cc1nc(-c2ccccc2)sc1C(=O)NNC(=S)Nc1ccccc1</smiles>

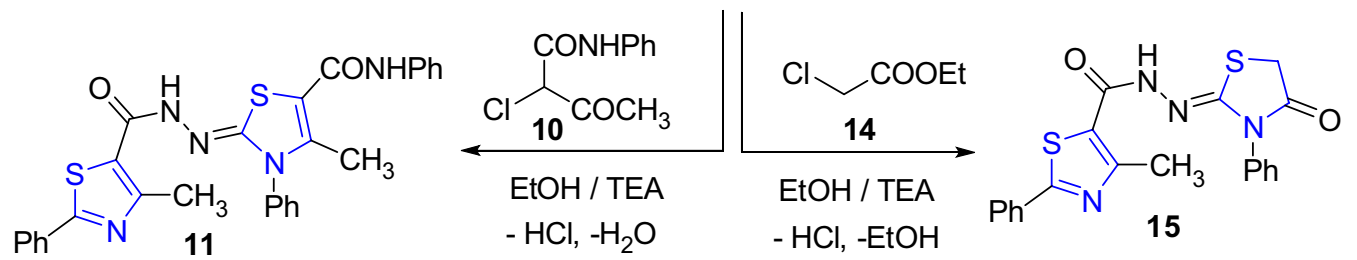

Scheme 3 Synthesis of thiazole derivatives $\mathbf{9}, \mathbf{1 1}, \mathbf{1 3}$ and $\mathbf{1 5}$

Table 1 The in vitro inhibitory activity of the tested compounds against tumor cell lines expressed as $I_{50}$ values $(\mu \mathrm{g} / \mathrm{mL}) \pm$ standard deviation from three replicates

\begin{tabular}{llll}
\hline $\begin{array}{l}\text { Tested } \\
\text { compounds }\end{array}$ & $\mathbf{I C}_{\mathbf{5 0}}(\boldsymbol{\mu g} / \mathbf{m L})$ & $\begin{array}{l}\text { Tested } \\
\text { compounds }\end{array}$ & $\mathbf{I C}_{\mathbf{5 0}}(\boldsymbol{\mu g} / \mathbf{m L})$ \\
\hline Cisplatin & $1.43 \pm 2.03$ & $\mathbf{7 c}$ & $7.51 \pm 0.64$ \\
$\mathbf{5 a}$ & $22.3 \pm 2.41$ & $\mathbf{9}$ & $17.4 \pm 0.73$ \\
$\mathbf{5 b}$ & $20.3 \pm 3.70$ & $\mathbf{1 1}$ & $1.98 \pm 1.22$ \\
$\mathbf{5 c}$ & $57.2 \pm 7.12$ & $\mathbf{1 3}$ & $35.1 \pm 10.8$ \\
$\mathbf{7 a}$ & $2.14 \pm 3.54$ & $\mathbf{1 5}$ & $3.31 \pm 2.65$ \\
$\mathbf{7 b}$ & $1.61 \pm 1.92$ & & \\
\hline
\end{tabular}

Lim. Stone, Staffordshire, UK). IR spectra were measured on PyeUnicamSP 3300 and Shimadzu FTIR 8101 PC infrared spectrophotometers (Shimadzu, Tokyo, Japan) in potassium bromide discs. NMR spectra were measured on a Varian Mercury VX-300 NMR spectrometer (Varian, Inc., Karlsruhe, Germany) operating at $300 \mathrm{MHz}$ $\left({ }^{1} \mathrm{HNMR}\right)$ and run in deuterated dimethylsulfoxide $\left(\right.$ DMSO- $\left.d_{6}\right)$. Chemical shifts were related to that of the solvent. Mass spectra were recorded on a Shimadzu GCMS-QP1000 EX mass spectrometer (Tokyo, Japan) at $70 \mathrm{eV}$. Elemental analyses were measured by using a German made Elementarvario LIII CHNS analyzer. 2-(4-Methyl-2-phenylthiazole-5-carbonyl)- $N$-phenylhydrazinecarbothioamide (3) [31], and hydrazonoyl halides 4a-c [32] were prepared as reported in the respective literature.

\section{Synthetic procedures}

\section{Synthesis of hydrazonoyl chlorides $5 a-c$}

A mixture of 4-methyl-2-phenylthiazole-5-carbohydrazide (2) (2.33 g, $10 \mathrm{mmol})$ and the appropriate hydrazonoyl chlorides $\mathbf{4 a}-\mathbf{c}(10 \mathrm{mmol})$ in ethanol $(30 \mathrm{~mL})$ was refluxed for $3-5 \mathrm{~h}$ (monitored through TLC).The resulting solid product was collected and recrystallized from the proper solvent to give the corresponding products 5a-c.

2-(2-(4-Methyl-2-phenylthiazole-5-carbonyl)hydrazono)$N^{\prime}$-phenylpropane hydrazonoyl chloride (5a) Yellow solid; yield (84\%); m.p. $188-190{ }^{\circ} \mathrm{C}(\mathrm{EtOH}) ; \mathrm{IR}(\mathrm{KBr})$ v 3440, $3316(2 \mathrm{NH}), 3036,2922(\mathrm{CH}), 1640(\mathrm{C}=\mathrm{O})$, $1599(\mathrm{C}=\mathrm{N}) \mathrm{cm}^{-1} ;{ }^{1} \mathrm{H}$ NMR (DMSO- $\left.d_{6}\right) \delta 2.36(\mathrm{~s}, 3 \mathrm{H}$, $\left.\mathrm{CH}_{3}\right), 2.76\left(\mathrm{~s}, 3 \mathrm{H}, \mathrm{CH}_{3}\right), 7.06-7.86(\mathrm{~m}, 10 \mathrm{H}, \mathrm{ArH}), 10.03$ (s, br, $1 \mathrm{H}, \mathrm{D}_{2} \mathrm{O}$-exchangeable $\left.\mathrm{NH}\right), 10.57(\mathrm{~s}, \mathrm{br}, 1 \mathrm{H}$, $\mathrm{D}_{2} \mathrm{O}$-exchangeable $\left.\mathrm{NH}\right) ; \mathrm{MS} \mathrm{m} / \mathrm{z}(\%): 413\left(\mathrm{M}^{+}+2,12\right)$, $411\left(\mathrm{M}^{+}, 40\right), 375$ (48), 202 (100), 174 (45), 71 (26). Anal. Calcd for $\mathrm{C}_{20} \mathrm{H}_{18} \mathrm{ClN}_{5} \mathrm{OS}$ (411.91): C, 58.32; H, 4.40; N, 17.00. Found: C, 58.19; H, 4.37; N, $16.88 \%$.

2-(2-(4-Methyl-2-phenylthiazole-5-carbonyl)hydrazono)$N^{\prime}$-(p-tolyl)propane- hydrazonoylchloride (5b) Yellow solid; yield (86\%); m.p. $172-174{ }^{\circ} \mathrm{C}(\mathrm{EtOH}) ; \mathrm{IR}(\mathrm{KBr}) \mathrm{v}$ 3437, $3313(2 \mathrm{NH}), 3041,2917(\mathrm{CH}), 1679(\mathrm{C}=\mathrm{O}), 1598$ $(\mathrm{C}=\mathrm{N}) \mathrm{cm}^{-1} ;{ }^{1} \mathrm{H}$ NMR (DMSO- $\left.d_{6}\right) \delta 2.24\left(\mathrm{~s}, 3 \mathrm{H}, \mathrm{CH}_{3}\right)$, 2.34 (s, 3H, $\left.\mathrm{CH}_{3}\right), 2.77\left(\mathrm{~s}, 3 \mathrm{H}, \mathrm{CH}_{3}\right), 7.08-7.99(\mathrm{~m}, 9 \mathrm{H}$, ArH), 10.06 (s, br, 1H, $\mathrm{D}_{2} \mathrm{O}$-exchangeable $\mathrm{NH}$ ), 10.59 (s, br, $1 \mathrm{H}, \mathrm{D}_{2} \mathrm{O}$-exchangeable $\left.\mathrm{NH}\right) ; \mathrm{MS} \mathrm{m} / \mathrm{z}(\%) 427\left(\mathrm{M}^{+}+2\right.$, 


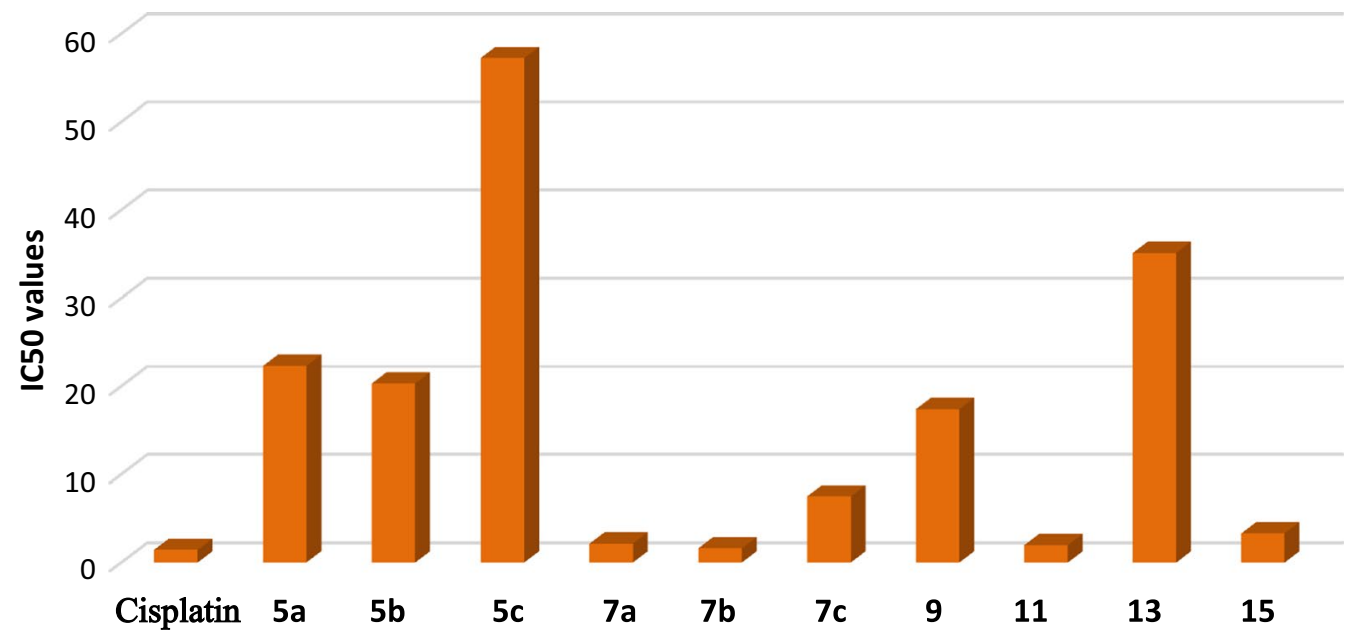

Fig. 3 Comparison of the $\mathrm{IC}_{50}$ of the new synthesized compounds against Cisplatin

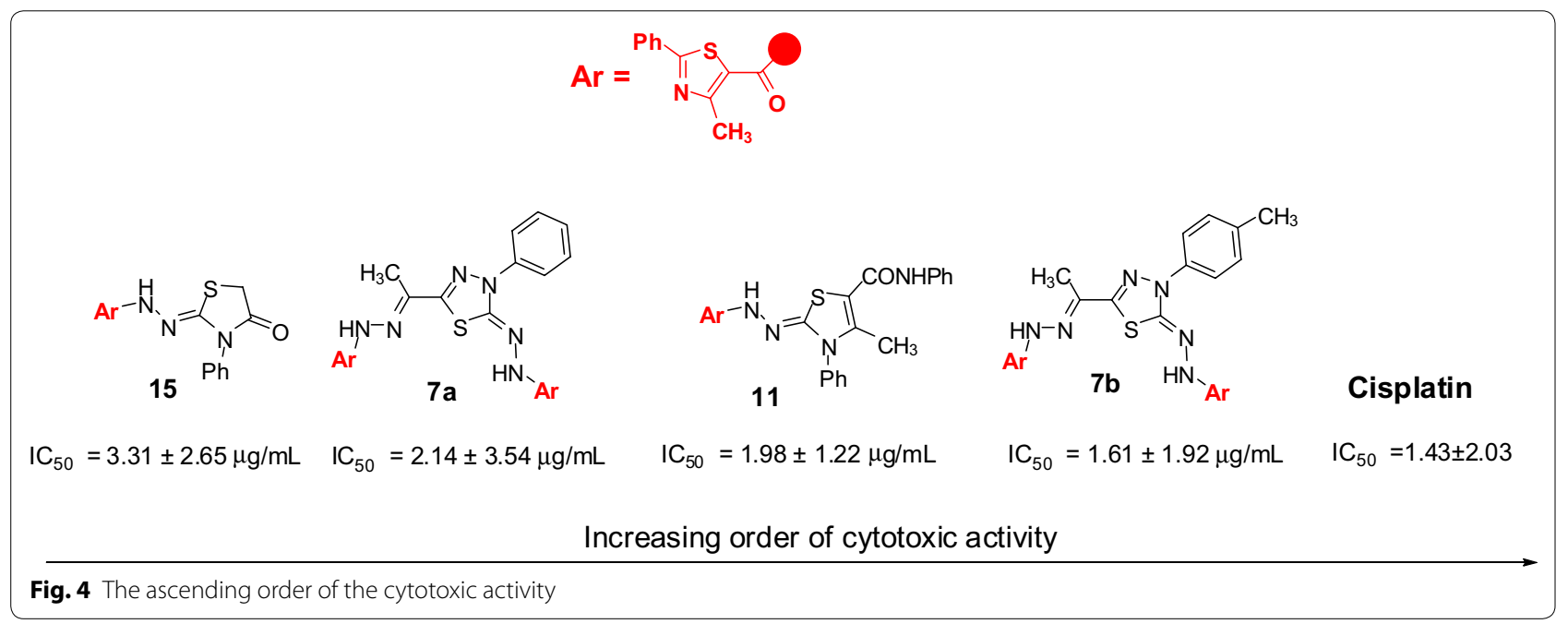

10), 425 ( $\left.\mathrm{M}^{+}, 33\right), 389$ (26), 202 (81), 106 (100), 64 (66). Anal. Calcd for $\mathrm{C}_{21} \mathrm{H}_{20} \mathrm{ClN}_{5} \mathrm{OS}$ (425.93): C, 59.22; H, 4.73; N, 16.44. Found: C, 59.18; H, 4.65; N, 16.37\%.

$N^{\prime}$-(4-Chlorophenyl)-2-(2-(4-methyl-2-phenylthiazole -5-carbonyl)hydrazono) propane hydrazonoyl chloride (5c) Yellow solid; yield (87\%); m.p. $194-196{ }^{\circ} \mathrm{C}$ (DMF); IR (KBr) v 3434, 3319 (2NH), 3044, $2926(\mathrm{CH}), 1682$ $(\mathrm{C}=\mathrm{O}), 1593(\mathrm{C}=\mathrm{N}) \mathrm{cm}^{-1} ;{ }^{1} \mathrm{H}$ NMR (DMSO-d $) \delta 2.37$ (s, 3H, $\left.\mathrm{CH}_{3}\right), 2.77\left(\mathrm{~s}, 3 \mathrm{H}, \mathrm{CH}_{3}\right), 7.08-7.99(\mathrm{~m}, 9 \mathrm{H}, \mathrm{Ar}-\mathrm{H})$, 10.06 (s, br, $1 \mathrm{H}, \mathrm{D}_{2} \mathrm{O}$-exchangeable $\left.\mathrm{NH}\right), 10.57$ (s, br, $1 \mathrm{H}$, $\mathrm{D}_{2} \mathrm{O}$-exchangeable $\left.\mathrm{NH}\right) ; \mathrm{MS} \mathrm{m} / \mathrm{z}(\%) 446\left(\mathrm{M}^{+}, 8\right), 283$ (14), 202 (39), 104 (46), 80 (100), 64 (90). Anal. Calcd for $\mathrm{C}_{20} \mathrm{H}_{17} \mathrm{Cl}_{2} \mathrm{~N}_{5} \mathrm{OS}$ (446.35): C, 53.82; $\mathrm{H}, 3.84 ; \mathrm{N}, 15.69$. Found: C, 53.75; H, 3.79; N, 15.58\%.

\section{Synthesis of 1,3,4-thiadiazole derivatives 7a-c}

A mixture of compound $3(0.368 \mathrm{~g}, 1 \mathrm{mmol})$ and the appropriate hydrazonoyl chlorides $5 \mathbf{a}-\mathbf{c}(1 \mathrm{mmol})$ in ethanol $(20 \mathrm{~mL})$ containing triethylamine $(0.1 \mathrm{~g}, 1 \mathrm{mmol})$ was refluxed for $6 \mathrm{~h}$. The formed solid product was filtered, washed with methanol, dried and recrystallized from the suitable solvents to give corresponding products $7 \mathbf{a}-\mathbf{c}$.

4-Methyl-N'-(1-(-5-(2-(4-methyl-2-phenylthiazole-5carbonyl)hydrazono)-4-phenyl-4,5-dihydro-1,3,4-thiadiazol-2-yl)ethylidene)-2-phenylthiazole-5carbohydrazide(7a) Yellow solid; yield (74\%); m.p. $162-164{ }^{\circ} \mathrm{C}(\mathrm{EtOH}) ; \mathrm{IR}(\mathrm{KBr})$ v 3421, $3307(2 \mathrm{NH})$, 3031, $2951(\mathrm{CH}), 1649(\mathrm{C}=\mathrm{O}), 1596(\mathrm{C}=\mathrm{N}) \mathrm{cm}^{-1}$; 
${ }^{1} \mathrm{H}$ NMR (DMSO- $\left.d_{6}\right) \delta 2.34\left(\mathrm{~s}, 3 \mathrm{H}, \mathrm{CH}_{3}\right), 2.66(\mathrm{~s}, 3 \mathrm{H}$, $\left.\mathrm{CH}_{3}\right), 2.76\left(\mathrm{~s}, 3 \mathrm{H}, \mathrm{CH}_{3}\right), 6.97-8.14(\mathrm{~m}, 15 \mathrm{H}, \mathrm{ArH}), 10.18$ (s, br, $1 \mathrm{H}, \mathrm{D}_{2} \mathrm{O}$-exchangeable $\left.\mathrm{NH}\right), 11.17(\mathrm{~s}, \mathrm{br}, 1 \mathrm{H}$, $\mathrm{D}_{2} \mathrm{O}$-exchangeable $\left.\mathrm{NH}\right) ; \mathrm{MS} \mathrm{m} / \mathrm{z}(\%) 650\left(\mathrm{M}^{+}, 34\right), 526$ (30), 416 (60), 358 (28), 104 (55), 64 (100). Anal. Calcd for $\mathrm{C}_{32} \mathrm{H}_{26} \mathrm{~N}_{8} \mathrm{O}_{2} \mathrm{~S}_{3}(650.80): C, 59.06 ; \mathrm{H}, 4.03 ; \mathrm{N}, 17.22$. Found C, 58.94; H, 4.01; N, 17.07\%.

4-Methyl-N'-(1-(5-(2-(4-methyl-2-phenylthiazole-5-c arbonyl)hydrazono)-4-(p-tolyl)-4,5-dihydro-1,3,4-thiadiazol-2-yl)ethylidene)-2-phenylthiazole-5-carbohydrazide (7b) Yellow solid; yield (72\%); m.p. $149-151{ }^{\circ} \mathrm{C}$ (EtOH); IR (KBr) v 3422, $3328(2 \mathrm{NH}), 3053,2929(\mathrm{CH})$, $1647(\mathrm{C}=\mathrm{O}), 1597(\mathrm{C}=\mathrm{N}) \mathrm{cm}^{-1}$; ${ }^{1} \mathrm{H}$ NMR (DMSO$\left.d_{6}\right) \delta 2.26\left(\mathrm{~s}, 3 \mathrm{H}, \mathrm{CH}_{3}\right), 2.35\left(\mathrm{~s}, 3 \mathrm{H}, \mathrm{CH}_{3}\right), 2.65(\mathrm{~s}, 3 \mathrm{H}$, $\left.\mathrm{CH}_{3}\right), 2.76\left(\mathrm{~s}, 3 \mathrm{H}, \mathrm{CH}_{3}\right), 6.91-8.03(\mathrm{~m}, 14 \mathrm{H}, \mathrm{ArH}), 10.18$ $\left(\mathrm{s}, \mathrm{br}, 1 \mathrm{H}, \mathrm{D}_{2} \mathrm{O}\right.$-exchangeable $\left.\mathrm{NH}\right), 11.14(\mathrm{~s}, \mathrm{br}, 1 \mathrm{H}$, $\mathrm{D}_{2} \mathrm{O}$-exchangeable $\left.\mathrm{NH}\right) ; \mathrm{MS} \mathrm{m} / \mathrm{z}(\%) 664\left(\mathrm{M}^{+}, 35\right), 553$ (60), 334 (19), 202 (65), 104 (85), 64 (100). Anal. Calcd for $\mathrm{C}_{33} \mathrm{H}_{28} \mathrm{~N}_{8} \mathrm{O}_{2} \mathrm{~S}_{3}$ (664.82): C, 59.62; H, 4.25; N, 16.85. Found C, 59.47; H, 4.17; N, $16.79 \%$.

$N^{\prime}$-(3-(4-Chlorophenyl)-5-(1-(2-(4-methyl-2-phenylt hiazole-5-carbonyl)hydrazono)eth-yl)-1,3,4-thiadiazol-2(3H)-ylidene)-4-methyl-2-phenylthiazole-5-carbohydrazide (7c) Yellow solid; yield (76\%); m.p. 191-193 ${ }^{\circ} \mathrm{C}$ (Dioxane); IR (KBr) v 3424, $3312(2 \mathrm{NH}), 3047,2932(\mathrm{CH})$, $1649(\mathrm{C}=\mathrm{O}), 1599(\mathrm{C}=\mathrm{N}) \mathrm{cm}^{-1} ;{ }^{1} \mathrm{H}$ NMR (DMSO- $\left.d_{6}\right) \delta$ $2.33\left(\mathrm{~s}, 3 \mathrm{H}, \mathrm{CH}_{3}\right), 2.66\left(\mathrm{~s}, 3 \mathrm{H}, \mathrm{CH}_{3}\right), 2.77\left(\mathrm{~s}, 3 \mathrm{H}, \mathrm{CH}_{3}\right), 6.90-$ $8.11(\mathrm{~m}, 14 \mathrm{H}, \mathrm{ArH}), 10.13\left(\mathrm{~s}, \mathrm{br}, 1 \mathrm{H}, \mathrm{D}_{2} \mathrm{O}\right.$-exchangeable $\mathrm{NH}), 11.19$ (s, br, 1H, $\mathrm{D}_{2} \mathrm{O}$-exchangeable $\left.\mathrm{NH}\right) ; \mathrm{MS} \mathrm{m} / \mathrm{z}$ (\%) $686\left(\mathrm{M}^{+}+2,8\right), 684(\mathrm{M}+, 26), 513$ (53), 368 (39), 257 (17), 104 (25), 64 (100). Anal.Calcd for $\mathrm{C}_{32} \mathrm{H}_{25} \mathrm{ClN}_{8} \mathrm{O}_{2} \mathrm{~S}_{3}$ (685.24): C, 56.09; H, 3.68; N, 16.35. Found C, 56.02; H, $3.58 ; \mathrm{N}, 16.22 \%$.

\section{General procedure for the synthesis of thiazole derivatives 9 , 11,13 , and 15}

A mixture of compound $3(0.368 \mathrm{~g}, 1 \mathrm{mmol})$ and the appropriate $\alpha$-halo-compounds namely, 3 -chloropentane-2,4-dione (8), 2-chloro-3-oxo-N-phenylbutanamide (10), 2-bromo-1-phenyl ethanone (12) and ethyl 2-chloroacetate (14) (1 mmol for each) in ethanol $(20 \mathrm{~mL})$ containing triethylamine $(0.1 \mathrm{~g}, 1 \mathrm{mmol})$ was refluxed for 4-6 h. (monitored by TLC The solid product was filtered, washed with water, dried and recrystallized from the proper solvent to give the corresponding thiazole derivatives $9,11,13$, and 15 , respectively.

$N^{\prime}$-(5-Acetyl-4-methyl-3-phenylthiazol-2(3H)-ylidene)-4 -methyl-2-phenylthiazole-5-carbohydrazide (9) Yellow solid; yield (78\%); m.p. $155-157^{\circ} \mathrm{C}(\mathrm{EtOH}) ; \mathrm{IR}(\mathrm{KBr})$ v 3432
(NH), 3036, $2993(\mathrm{CH}), 1695,1648(2 \mathrm{C}=\mathrm{O}), 1590(\mathrm{C}=\mathrm{N})$ $\mathrm{cm}^{-1} ;{ }^{1} \mathrm{H}$ NMR(DMSO- $\left.d_{6}\right) \delta 2.32\left(\mathrm{~s}, 3 \mathrm{H}, \mathrm{CH}_{3}\right), 2.46(\mathrm{~s}, 3 \mathrm{H}$, $\left.\mathrm{CH}_{3}\right), 2.77$ (s, 3H, $\left.\mathrm{CH}_{3}\right), 6.91-7.86(\mathrm{~m}, 10 \mathrm{H}, \mathrm{ArH}), 10.61$ (s, br, $1 \mathrm{H}, \mathrm{D}_{2} \mathrm{O}$-exchangeable $\left.\mathrm{NH}\right) ; \mathrm{MS} \mathrm{m} / \mathrm{z}(\%) 448\left(\mathrm{M}^{+}\right.$, 57), 246 (60), 176 (35), 104 (80), 77 (100). Anal.Calcd for $\mathrm{C}_{23} \mathrm{H}_{20} \mathrm{~N}_{4} \mathrm{O}_{2} \mathrm{~S}_{2}$ (448.56): C, 61.59; H, 4.49; N, 12.49. Found C, 61.48; H, 4.36; N, 12.37\%.

4-Methyl-2-(2-(4-methyl-2-phenylthiazole-5-carbonyl) hydrazono)-N-3-diphenyl-2,3-dihydrothiazole-5-carboxamide (11) Yellow solid; yield (79\%); m.p. $182-84{ }^{\circ} \mathrm{C}$ (DMF); IR (KBr): v 3435, $3176(2 \mathrm{NH}), 3030,2928(\mathrm{CH})$, $1671,1649(2 \mathrm{C}=\mathrm{O}), 1594(\mathrm{C}=\mathrm{N}) \mathrm{cm}^{-1} ;{ }^{1} \mathrm{H}$ NMR (DMSO$\left.d_{6}\right) \delta 2.36\left(\mathrm{~s}, 3 \mathrm{H}, \mathrm{CH}_{3}\right), 2.76\left(\mathrm{~s}, 3 \mathrm{H}, \mathrm{CH}_{3}\right), 6.97-7.73(\mathrm{~m}$, $15 \mathrm{H}, \mathrm{ArH}), 10.46$ (s, br, $1 \mathrm{H}, \mathrm{D}_{2} \mathrm{O}$-exchangeable $\left.\mathrm{NH}\right), 11.72$ (s, br, $1 \mathrm{H}, \mathrm{D}_{2} \mathrm{O}$-exchangeable $\left.\mathrm{NH}\right) ; \mathrm{MS} \mathrm{m} / \mathrm{z}(\%) 525\left(\mathrm{M}^{+}\right.$, 7), 447 (16), 334 (100), 200 (59), 77 (89). Anal.Calcd for $\mathrm{C}_{28} \mathrm{H}_{23} \mathrm{~N}_{5} \mathrm{O}_{2} \mathrm{~S}_{2}$ (525.64): C, 63.98; $\mathrm{H}, 4.41 ; \mathrm{N}, 13.32$. Found C, 63.84; H, 4.30; N, 13.28\%.

$N^{\prime}$-(3,4-Diphenylthiazol-2(3H)-ylidene)-4-methyl-2-ph enylthiazole-5-carbohydrazide (13) Yellow solid; yield (70\%); m.p. $174-178{ }^{\circ} \mathrm{C}(\mathrm{EtOH}) ; \mathrm{IR}(\mathrm{KBr})$ v $3369(\mathrm{NH})$, 3047, 2926(CH), $1648(\mathrm{C}=\mathrm{O}), 1594(\mathrm{C}=\mathrm{N}) \mathrm{cm}^{-1} ;{ }^{1} \mathrm{H}$ NMR (DMSO- $\left.d_{6}\right) \delta 2.75\left(\mathrm{~s}, 3 \mathrm{H}, \mathrm{CH}_{3}\right), 7.03(\mathrm{~s}, 1 \mathrm{H}$, thiazole-H5), 7.35-8.02 (m, 15H, ArH), 10.73 (s, br, 1H, $\mathrm{D}_{2} \mathrm{O}$-exchangeable $\left.\mathrm{NH}\right) ; \mathrm{MS} \mathrm{m} / \mathrm{z}(\%) 468\left(\mathrm{M}^{+}, 25\right)$, 334 (100), 200 (40), 104 (69), 64(65). Anal.Calcd for $\mathrm{C}_{26} \mathrm{H}_{20} \mathrm{~N}_{4} \mathrm{OS}_{2}$ (468.59): C, 66.64; H, 4.30; N, 11.96. Found C, 66.55; H, 4.21; N, $11.79 \%$.

4-Methyl-N'-(4-oxo-3-phenylthiazolidin-2-ylidene)-2-p henylthiazole-5-carbo- hydrazide (15) Yellowish-white solid; yield (72\%); m.p. $192-194{ }^{\circ} \mathrm{C}$ (Dioxane); IR ( $\left.\mathrm{KBr}\right)$ v 3331(NH), 3036, $2926(\mathrm{CH}), 1726,1648(2 \mathrm{C}=\mathrm{O}), 1596$ $(\mathrm{C}=\mathrm{N}) \mathrm{cm}^{-1} ;{ }^{1} \mathrm{H}$ NMR (DMSO- $\left.d_{6}\right) \delta 2.65\left(\mathrm{~s}, 3 \mathrm{H}, \mathrm{CH}_{3}\right)$, $4.23\left(\mathrm{~s}, 2 \mathrm{H}\right.$, thiazolone- $\left.\mathrm{CH}_{2}\right), 7.40-7.88(\mathrm{~m}, 10 \mathrm{H}, \mathrm{ArH})$, 10.82 (s, br, $1 \mathrm{H}, \mathrm{D}_{2} \mathrm{O}$-exchangeable $\mathrm{NH}$ ); $\mathrm{MS} \mathrm{m} / \mathrm{z}$ (\%) 408 $\left(\mathrm{M}^{+}, 65\right)$, 334 (18), 202 (100), 104 (86), 64 (69). Anal.Calcd for $\mathrm{C}_{20} \mathrm{H}_{16} \mathrm{~N}_{4} \mathrm{O}_{2} \mathrm{~S}_{2}$ (408.50): C, 58.80; H, 3.95; N, 13.72 . Found C, 58.68; H, 3.84; N, 13.64\%.

\section{Anticancer activity}

The cytotoxic evaluation of the synthesized compounds was carried out at the Regional Center for Mycology and Biotechnology at Al-Azhar University, Cairo, Egypt according to the reported method [33].

\section{Conclusions}

We successfully synthesized a series of novel heterocycles containing thiazole and 1,3,4-thiadiazole rings by a facile and convenient method. The structure of the newly 
prepared compounds was established based on both elemental analysis and spectroscopic data. The anticancer activity of the synthesized compounds was measured and showed promising activity.

\section{Abbreviations}

HepG2: human hepatocellular carcinoma; EtOH: ethanol; m.p.: melting point; TEA: triethylamine; IR: infra-red; ATCC: American Type Culture Collection; TLC: thin layer chromatography.

\section{Authors' contributions}

SMG, NAK and YNM carried the literature study and designed synthetic schemes, MRA and SA contributed in the synthesis and purification of the compounds. All authors read and approved the final manuscript.

\section{Author details \\ 1 Department of Chemistry, Faculty of Science, Cairo University, Giza 12613, Egypt. ${ }^{2}$ Department of Pharmaceutical Chemistry, Faculty of Pharmacy, MIU University, Cairo, Egypt. ${ }^{3}$ Department of Pharmaceutical Chemistry, Faculty of Pharmacy, King Khalid University, Abha 61441, Saudi Arabia. ${ }^{4}$ Department of Chemistry, Faculty of Science, University of Beni Suef, Beni Suef, Egypt. ${ }^{5}$ Department of Chemistry, College of Science, King Saud University, P. O. Box 2455, Riyadh 11451, Saudi Arabia.}

\section{Acknowledgements}

The authors extend their sincere appreciation to the Deanship of Scientific Research at the King Saud University for its funding this Prolific Research group (PRG-1437-29).

\section{Competing interests}

The authors declare that they have no competing interests.

\section{Publisher's Note}

Springer Nature remains neutral with regard to jurisdictional claims in published maps and institutional affiliations.

Received: 16 July 2017 Accepted: 10 October 2017

Published online: 16 October 2017

\section{References}

1. Karegoudar P, Karthikeyan MS, Prasad DJ, Mahalinga M, Holla BS, Kumari NS (2008) Synthesis of some novel 2,4-disubstituted thiazoles as possible antimicrobial agents. Eur J Med Chem 43:261-267

2. Gomha SM, Kheder NA, Abdelaziz MR, Mabkhot YN, Alhajoj AM (2017) A facile synthesis and anticancer activity of some novel thiazoles carrying 1,3,4-thiadiazole moiety. Chem. Central J 11:25

3. Gomha SM, Abdel-Aziz HA (2012) Synthesis of new heterocycles derived from 3-(3-methyl-1H-indol-2-yl)-3-oxopropanenitrile as potent antifungal agents. Bull Kor Chem Soc 33:2985-2990

4. Kheder NA, Riyadh SM, Asiry AM (2013) Azoles and bis-Azoles: synthesis and biological evaluation as antimicrobial and anti-cancer agents. Chem Pharm Bull 61:504-510

5. Sharma PK, Sawhney SN, Gupta A, Singh GB, Bani S (1998) Synthesis and antiinflammatory activity of some 3-(2-thiazolyl)-1,2-benzisothiazoles. Indian J Chem 37B:376-381

6. Shih MH, Ying KF (2004) Syntheses and evaluation of antioxidant activity of sydnonyl substituted thiazolidinone and thiazoline derivatives. Bioorg Med Chem 12:4633-4643

7. Shiradkar M, Kumar GVS, Dasari V, Tatikonda S, Akula KC, Shah R (2007) Clubbed triazoles: a novel approach to antitubercular drugs. Eur J Med Chem 42:807-816

8. Gomha SM, Khalil KD (2012) A convenient ultrasound-promoted synthesis and cytotoxic activity of some new thiazole derivatives bearing a coumarin nucleus. Molecules 17:9335-9347

9. Gomha SM, Salah TA, Abdelhamid AO (2015) Synthesis, characterization and pharmacological evaluation of some novel thiadiazoles and thiazoles incorporating pyrazole moiety as potent anticancer agents. Monatsh Chem 146:149-158

10. Gomha SM, Riyadh SM, Abbas IM, Bauomi MA (2013) Synthetic utility of ethylidenethiosemicarbazide: synthesis and anti-cancer activity of 1,3-thiazines and thiazoles with imidazole moiety. Heterocycles 87:341-356

11. Gomha SM, Salah TA, Hassaneen HME, Abdel-aziz H, Khedr MA (2016) Synthesis, characterization and molecular docking of novel bioactive thiazolyl-thiazole derivatives as promising cytotoxic antitumor drug. Molecules 21:1-17

12. Gomha SM, Abdelaziz MR, Abdel-aziz HM, Hassan SA (2017) Green synthesis and molecular docking of thiazolyl-thiazole derivatives as potential cytotoxic agents. MiniRev Med Chem 17:805-815

13. Oruç EE, Rollas S, Kandemirli F, Shvets N, Dimoglo AS (2004) 1,3,4-thiadiazole derivatives. Synthesis, structure elucidation, and structure-antituberculosis activity relationship investigation. J Med Chem 47:6760-6767

14. Dawood KM, Abdel-Gawad H, Rageb EA, Ellithey M, Mohamed HA (2006) Synthesis, anticonvulsant, and anti-inflammatory evaluation of some new benzotriazole and benzofuran-based heterocycles. Bioorg Med Chem 14:3672-3680

15. Clerici F, Pocar D, Guido M, Loche A, Perlini V, Brufani M (2001) Synthesis of 2-amino-5-sulfanyl-1,3,4-thiadiazole derivatives and evaluation of their antidepressant and anxiolytic activity. J Med Chem 44:931-936

16. Foroumadi A, Kiani Z, Soltani F (2003) Antituberculosis agents VIII. Synthesis and in vitro antimycobacterial activity of alkyl alpha-[5-(5-nitro2-thienyl)-1,3,4-thiadiazole-2-ylthio] acetates. Farmaco 58:1073-1076

17. Mullick P, Khan SA, Verma S, Alam O (2011) Thiadiazole derivatives as potential anticonvulsant agents. Bull Kor Chem Soc 32:1011-1016

18. Gomha SM, Kheder NA, Abdelhamid AO, Mabkhot YN (2016) One pot single step synthesis and biological evaluation of some novel bis(1,3,4thiadiazole) derivatives as potential cytotoxic agents. Molecules 21:1532

19. Gomha SM, Abdel-aziz HM, Khalil KD (2016) Synthesis and SAR study of the novel thiadiazole-imidazole derivatives as new anti-cancer agents. Chem Pharm Bull 64:1356-1363

20. Gomha SM, Badrey MG, Edrees MM (2016) Heterocyclization of a bis-thiosemicarbazone of 2,5-diacetyl-3,4-disubstituted-thieno[2,3-b]thiophene bis-thiosemicarbazones leading to bis-thiazoles and bis-1,3,4-thiadiazoles as anti-breast cancer agents. J Chem Res 40:120-125

21. Gomha SM (2009) A facile one-pot synthesis of 6,7,8,9-tetrahydrobenzo [4,5]thieno [2,3-d]-1,2,4-triazolo[4,5-a]pyrimidin-5-ones. Monatsh Chem 140:213-220

22. Abdelhamid AO, Gomha SM, Abdelriheem NA (2017) Utility of 2-(5-methyl-1-phenyl-1H-pyrazol-4-yl)-2-oxo- $\mathrm{N}^{\prime}$-phenylaceto-hydrazonoyl bromide as precursor for synthesis of new functionalized heterocycles. Synth Commun 47:999-1005

23. Abbas IM, Gomha SM, Elneairy MAA, Elaasser MM, Mabrouk BKA (2015) Synthesis and characterization of some novel fused thiazolo[3,2-a]pyrimidinones and pyrimido[2,1-b][1,3]thiazinones. J Chem Res 39:719-723

24. Gomha SM, Riyadh SM (2009) Synthesis of triazolo[4,3-b][1,2,4,5]tetrazines and triazolo[3,4-b][1,3,4]thiadiazines using chitosan as ecofriendly catalyst under microwave irradiation. Arkivoc 11:58-68

25. Abdallah MA, Riyadh SM, Abbas IM, Gomha SM (2005) Synthesis and biological activities of 7-arylazo-7H-pyrazolo[5,1-c][1,2,4]triazolo-6(5H)ones and 7-arylhydrazono-7H-[1, 2, 4]triazolo[3,4-b][1,3,4]thiadiazines. J Chin Chem Soc 52:987-994

26. Gomha SM, Badrey MG, Abdalla MM, Arafa RK (2014) Novel Anti-HIV-1 NNRTIs Based on a pyrazolo[4,3-d]isoxazole backbone scaffold: design, synthesis and exploration of molecular basis of action. Med Chem Commun 5:1685-1692

27. Abbas IM, Riyadh SM, Abdallah MA, Gomha SM (2006) A novel route to tetracyclic fused tetrazines and thiadiazines. J Heterocycl Chem 43:935-942

28. Dawood KM, Gomha SM (2015) Synthesis and anti-cancer activity of 1,3,4-thiadiazole and 1,3-thiazole derivatives having 1,3,4-oxadiazole moiety. J Heterocycl Chem 52:1400-1405

29. Gomha SM, Riyadh SM (2014) Multicomponent synthesis of novel pentaheterocyclic ring systems incorporating benzopyranopyridines scaffold. Synthesis 46:258-262

30. Gomha SM, Riyadh SM (2015) Cellulose sulfuric acid as an eco-friendly catalyst for novel one-pot synthesis of pyrido[2,3-d][1,2,4] triazolo[4,3-a] pyrimidin-5-ones. J Braz Chem Soc 26:916-923 
31. Tiperciuc B, Zaharia V, Colosi I, Moldovan C, Crişan O, Pîrnau A, Vlase L, Duma M, Oniga O (2012) Synthesis and evaluation of antimicrobial activity of some new hetaryl-azoles derivatives obtained from 2-aryl-4-methylthiazol-5-carbohydrazides and isonicotinic acid hydrazide. J Heterocycl Chem 49:1407-1414

32. Shawali AS, Gomha SM (2000) A New entry for short and regioselective synthesis of $[1,2,4]$ triazolo[4,3-b][1,2,4]-triazin-7(1H)-one. Adv Synth Catal 342:599-604
33. Gomha SM, Riyadh SM, Mahmmoud EA, Elasser MM (2015) Synthesis and anticancer activities of thiazoles, 1,3-thiazines, and thiazolidine using chitosan-grafted-poly(vinyl pyridine) as basic catalyst. Heterocycles 91:1227-1243

\section{Submit your manuscript to a SpringerOpen ${ }^{\circ}$ journal and benefit from:}

- Convenient online submission

- Rigorous peer review

- Open access: articles freely available online

- High visibility within the field

- Retaining the copyright to your article

Submit your next manuscript at $\boldsymbol{\nabla}$ springeropen.com 